\title{
Medicine Soaring with Nanofever
}

Chawla $\mathbf{R}^{1^{*}}$, Shetty $\mathrm{KS}^{2}$, Goyal $\mathbf{M}^{3}$, Rathore APS ${ }^{2}$ and Sharma $\mathrm{A}^{3}$

${ }^{1}$ Department of Orthodontics and Dentofacial Orthopedics, Penang International Dental College, Penang, Malaysia

${ }^{2}$ Department of Orthodontics and Dentofacial Orthopedics, Bapuji Dental College and Hospital, Davangere, Karnataka, India

${ }^{3}$ Department of Orthodontics and Dentofacial Orthopedics, Kothiwal Dental College and Research Centre, Moradabad, Uttar Pradesh, India

*Corresponding author: Dr. Riddhi Chawla, Department of Orthodontics and Dentofacial Orthopedics, Penang International Dental College, Penang , Malaysia, Tel: +91 7406552501; E-mail: riddhichawla@yahoo.co.in

Received date: December 28, 2017; Accepted date: February 22, 2018; Published date: March 1, 2018

Copyright: (C) 2018 Chawla R, et al. This is an open-access article distributed under the terms of the Creative Commons Attribution License, which permits unrestricted use, distribution, and reproduction in any medium, provided the original author and source are credited.

\begin{abstract}
Nanotechnology is the science related to understanding matter at approximately 1 to 100 nanometers. However, nanotechnology is not merely working with matter at the Nano scale, but also research and development of materials, devices, and systems that have novel properties and functions due to their Nano scale dimensions or components. It has not left any branch untouched be it medicine, dentistry or to be precise it exists all around us. Hence, the need today is to comprehend their properties to be able to control them before the reverse happens. Thus, it represents a perfect paradox challenging its judicious use to shape our future-Good or bad.
\end{abstract}

Keywords: Nanotechnology; Nanomedicine; Nanobots; Chlorophyll; Fullerenes

\section{Introduction}

"Nano" is a Greek word for DWARF whereby 1 nanometer $=1.0 \times$ $10^{-9}$ meter. Nanotechnology is the science related to understanding matter at approximately 1 to 100 nanometers. However, nanotechnology is not merely working with matter at the Nano scale, but also research and development of materials, devices, and systems that have novel properties and functions due to their Nano scale dimensions or components [1]. It exists naturally as Chloroplasts which contain Nano scale chlorophyll that converts light and carbon dioxide into biochemical energy or even as a mollusk, the Abalone [2]. Since we are surrounded by this mushrooming nanotechnology all around there is a need today to understand it closely. This review article attempts to elaborate how it has encroached on our medical technology today beginning from its birth, basis and progression.

\section{Science Behind Nanotechnology}

\section{The versatile carbon}

Carbon atoms are able to form 4 covalent bonds. It can bond with up to 4 other atoms and also with itself. It can form single, double or even triple bonds. It is capable of forming chains and various other shapes too which is a unique property.

\section{Forms of carbon fullerene}

Fullerenes are a family of carbon molecules composed entirely of carbon, in various shapes. They can exist as spherical Bucky balls (Figure 1) (a sphere made up of 60 carbons) or cylindrical Bucky tubes (Figure 2) (Width - few nanometers, Length - less than a micrometer to few millimeters) and possess unusual properties such as high tensile strength, high electrical conductivity, high ductility, high resistance to heat and relative chemical inactivity [2].

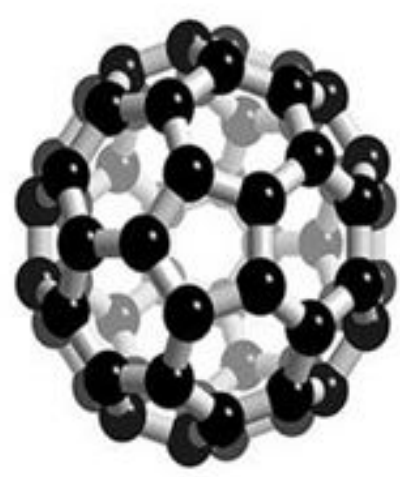

Figure 1: Bucky ball: A sphere made up of upto 60 carbons.

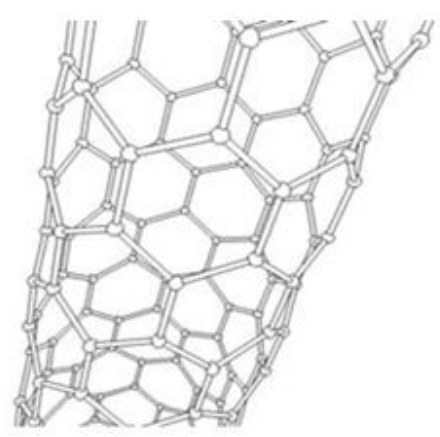

Figure 2: Buckytube: A cylindrical carbon fullerene with variable width and length. 


\section{Graphene: the wonder material}

Graphene is a two-dimensional monolayer of carbon atoms arranged in a honeycomb structure. Soon after its isolation in 2004 by Novoselov and Geim, it became the most studied material due to its extraordinary electronic properties.

Several relativistic quantum mechanical effects, such as the unusual half-integer quantum Hall effect, the Klein tunneling, the minimum conductivity or the Veselago lensing, arise from the relativistic massless nature of charge carriers in graphene, which are described by the Dirac equation.

Graphene has been traditionally considered hydrophobic, but recent studies have challenged this belief. Indeed, it has been shown that graphene may significantly modify the wettability when placed on superhydrophilic and superhydrophobic substrates [3]. The versatile properties of this wonder material are exploited for our benefit in a number of ways.

\section{What Makes Nano Level Different?}

Particles smaller than $50 \mathrm{~nm}$ do not obey the laws of classical physics rather they abide the quantum physics [2]. Thus they distinguish themselves optically, magnetically or even electrically.

Smaller the object, larger is the proportion of their constituent atoms near the surface; due to this researchers are able to "dial-in" many properties like melting temperature, magnetic properties, and color-that previously were impossible to obtain for a particular material by doping of fullerenes [4].

\section{Generations in Nano Products}

\section{Passive nanostructures (2000-2005)}

These products utilize the passive properties of nanomaterials including Nano layers which remain static once encapsulated eg, Titanium dioxide in sunscreen and carbon nanotubes in tennis racket.

\section{Active nanostructures (2005-2010)}

These actively respond in a predicable way to the environment around them, thus they change their state during use eg. Nanoparticles killing cancer cells.

\section{Systems of nano systems (2010-2015)}

Here an assembly of Nano structures work together to achieve a final goal eg. nano linked Proteins making up batteries, small nano electromechanical devices could search out cancer cells and turn off their reproductive capacity.

\section{Molecular nano systems (2015-2020)}

This stage involves the intricately designed atomic devices, leading to "unprecedented understanding and control over the basic building blocks of all natural and man-made things."

At this stage a single product will integrate a wide variety of capacities including independent power generation, information processing and communication, and mechanical operation.

\section{The singularity (2020 and beyond)}

It's a point on the exponential curve where the growth rate becomes almost infinite. Beyond it, human society completely reorganizes into an incomparable structure. The risk of artificial intelligence making discoveries beyond human comprehension will dawn [5-7].

\section{Nanoparticles}

\section{Quantum dots}

Nanoparticles such as quantum dot are the size of a protein molecule or a short stretch of DNA. Quantum dots can be engineered to absorb and emit many wavelengths of light with very sharp precision (Figure 3) [4].

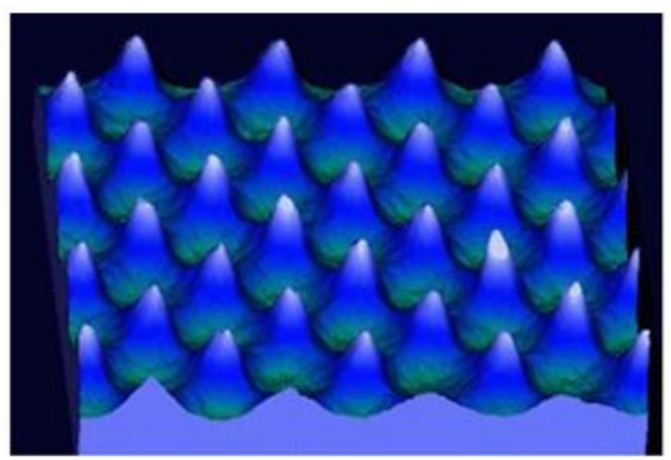

Figure 3: Nanodots/quantum dots: a nanoparticle of the size of a protein molecule.

\section{Dendrimers}

Dendrimers are nano branched synthetic molecules (Figure 4). They form one layer at a time so the size of the dendrimer is determined by the number of synthetic steps. Each dendrimer is usually only a few nanometers wide. The outside layer can be engineered to be composed of specific functional groups that can act as attachment to specifically bind other molecules.

Dendrimers may help in gene therapy for delivering DNA without triggering an immune response [8]. Unlike traditional polymers, dendrimers have received considerable attention in biological applications due to their high water solubility, biocompatibility, polyvalency and precise molecular weight. These features make them an ideal carrier for drug delivery and targeting applications.

Recently, a dendrimer based prodrug has been developed for paclitaxel (P-gp efflux substrate) that has focused on enhancement of permeability and transportation of drug across cellular barriers. The site specific delivery of the drug could be achieved by surface modification of dendrimers employing various targeting moieties such as folic acid (FA), peptides, monoclonal antibodies and sugar groups [9]. 
Citation: Chawla R, Shetty KS, Goyal M, Rathore APS, Sharma A (2018) Medicine Soaring with Nanofever. J Nanosci Curr Res 3: 220. doi:

Page 3 of 8

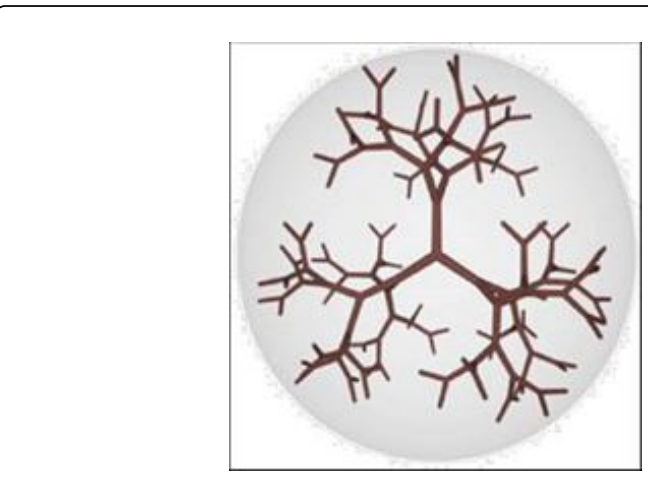

Figure 4: Dendrimer: a branched nanoparticle.

\section{Nanorods}

These are rod-shaped particles possessing diameter from 15-50 nm (Figure 5). They have a potential application as drug carriers, active sites to bind with specific biological tissue etc.

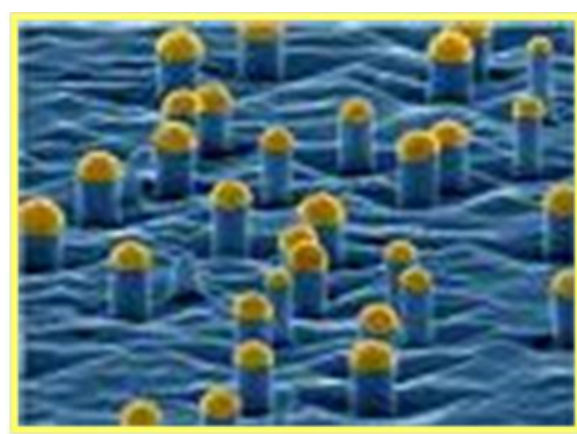

Figure 5: Nanorods: rod shaped nanoparticles.

\section{Nanotubes}

These are all-carbon nanotubes possessing diameter of 1.2-2 nm which can be used for new structural materials that are stronger and lighter ranging from supercomputers to new drug delivery systems (Figure 6).

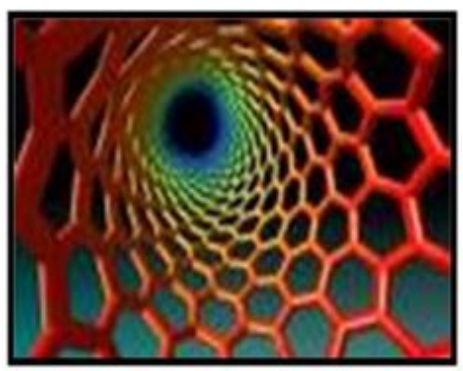

Figure 6: Nanotube: all carbon tubular nano structures.

\section{Nanopores}

These are about $20 \mathrm{~nm}$ in diameter encapsulated into silicon wafers (Figure 7). This way 'pig pancreatic cells' can be microencapsulated and isolated from foreign cells and hence replace nonfunctional islets of Langerhans cells in the pancreas. They can be implanted underneath the human skin without the need of immunosuppressants which put diabetic patients at a risk of infection [10].

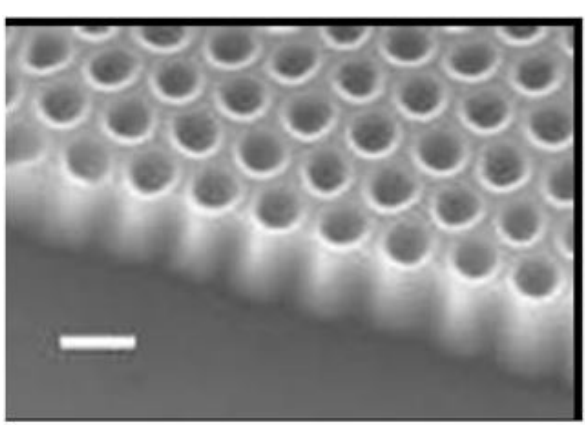

Figure 7: Nanopores: nanoparticles of about $20 \mathrm{~nm}$ diameter encapsulated into silicon wafers.

\section{Nanorobots}

\section{Design of nanorobots}

It is derived from biological models typically, $650 \mathrm{~nm}$ in length and $160 \mathrm{~nm}$ in diameter. Comprised of molecular sorting rotors (contact sensors), Robot arm (telescoping manipulator), fins and propellers (Figure 8) [11]. The outer shell is made up of strong chemically inert diamond. The acoustic relay attached to an onboard computer sends and receives ultrasound to communicate with medical team. Sensors and manipulators detect illnesses and perform cell by cell surgery. Pumps on the other hand remove toxins from the body and dispense drugs [12]. Medical Nano robots might utilize specific motility mechanisms in the human body tissues and navigate while acquiring energy and manipulate their surroundings by cytopenetration. An onboard Nano computer controls these functions [13].

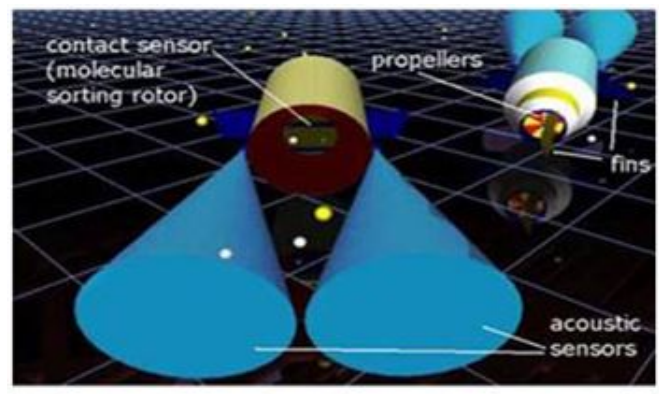

Figure 8: Parts of a nanorobot. 


\section{Nanomedicine}

\section{Applications in diagnosis and testing}

Nanoprobes: They are said to conquer the blood brain barrier and help in brain imaging. They provide a unique opportunity for rapid delivery of active compounds and can be used to cross the blood brain barrier (BBB) which represents a major barricade for a lot of chemical entities. The BBB is formed by endothelial cells surrounding the brain blood capillaries which are connected by tight junctions. Predominantly due to their size, nanoparticles can pass through the endothelial cell membrane [14].

Raman spectroscopy/microscopy imaging with surface enhanced Raman scattering (SERS) nanoparticles allows scientists to detect biological events in living cells or organisms in real time and with high sensitivity. The photoacoustic imaging has emerged as a hybrid of optical and ultrasound imaging for sensitive and quantitative tumor detection [15].

Quantum dots: Quantum dots can be engineered to absorb and emit many wavelengths of light with very sharp precision. This makes them ideal for protein-protein interaction studies as they can be linked to molecules to form long-lived probes. They can track biological events by tagging specific proteins or DNA in order to follow their progress through biological pathways. In medicine, quantum dots could be used for diagnostic purposes. Quantum dots (nanoparticles with properties such as size-tunable light emission), can produce exceptional images of tumor sites $[7,16]$.

Detection of clots: Nanoparticles move through the body of mice after being injected, and when they come across thrombin, the thrombin splits the peptides at a specific location, which releases particles that come out in urine [17]. Thus Nanotechnology urine test could detect deadly blood clots in humans too.

Snap shots of living system: The hypothetical autonomous molecular machines can explore and analyze living system including the human body in greater detail than ever before [18]. These snapshots would enable us to acquire a map of human body system more detailed than any we can currently imagine [19]. These machines could enter the body, take tissue samples and bring them out of the body for analysis.

Confocal microscope: Confocal microscopy coupled with fluorescent probes allowed determination of the path of some molecules in the cell in real time [18]. This microscope eliminates all out of focus rays as the excitation light is focused at diffraction limited spot of the sample. Its benefit is the possibility to generate images with contrast since only light from the focal plane is recorded [20].

Monitoring blood sugar level: Nanotechnology 'tattoos' are in development to help diabetics track glucose levels wherein, sensors are injected using a minimally invasive technology [21]. The intra and extra cellular Nano sensors reside under the skin and a portable imaging device is used to interrogate sensor as needed $[22,23]$.

Virtual biopsy: To get chair side imaging of tissues by alleviating discomfort of endoscope insertion is the future of diagnostic endoscopy. This can be done through virtual endoscopes or confocal microscope endoscope which make on-the-spot "virtual biopsies" and "virtual pathologic examinations" possible, requiring no biopsy, using an endoscope equipped with an ultra-compact spectroscope with MEMS on its tip among others [18]. Thus very soon endoscopic surgical treatments can be expected, such as in endoscopic fetal surgery and endoscopic brain surgery, as well as further coordination with navigation technologies and surgery support systems to allow safe execution of these procedures.

Video pills: The hypothetical Nano machines could be programmed to carry molecular sized digital video cameras that would transmit back to us a genuine inside view of the human body from a vantage point within a molecule or cell [18]. When swallowed it would send the images of gut from inside to the portable recorder outside which can be viewed on the computer.

Gastrobots, bone robots and nanoscouts: These hypothetical Nano robots when swallowed reach designated sites like the stomach lining, human bone marrow and travel through the blood vessels finding pathology with their sensors [24].

Cerenkov luminescence imaging: A probe for positron emission tomography (PET), which served as an optical biomaterial emitting Cerenkov photons, to in vivo optical imaging of tumor apoptosis, in order to evaluate early response to chemotherapy of drug-resistant gastric cancer has been tested wherein Vincristine-resistance of SGC7901/VCR and apoptosis-induction ability of vincristine and cisplatin were verified. Thus, CLI has proved to be a promising technique for in vivo imaging of apoptosis with radiopharmaceuticallabeled biomaterials [25].

\section{Infection management}

Various models have been postulated like pathogen rustler, T4 bacteriophage, Chrysalis, Parasite hunter 1, 2, 3, 4 and 5, Bug crunchers, Nano submarine, Molecular repair vessel, Artery cleaner, Lung cleaner, Nanotech playhouse 1,2,3,4 and 5, Virus Finders, Peeper, Stinger, driller, Dockers, mobile cell repairers, Transbots, Probots and destrobots which are equipped with optical Nano sensors and grasp foreign particles, virus, sick $\mathrm{T}$ lymphocytes and even drill tumor masses while travelling through the bloodstream [24].

A hydrogelator comprised of ibuprofen (IPF) and GFFY peptide linked through a cleavable ester bond has been tested. The synthesized hydrogelator could spontaneously self-assemble into a hydrogel under a heating-cooling process. When the hydrogel was acted upon by an esterase, IPF was released in a sustained manner. Moreover, the hydrogel had significantly elevated anti-inflammatory efficacy, compared with IPF, in RAW264.7 macrophages. The hydrogel showed good cytocompatibility, as well as excellent ocular biocompatibility when instilled topically. In vivo results further demonstrated that the hydrogel had therapeutic efficacy comparable to that of a current treatment, sodium diclofenac (DIC) eye drops, in suppressing ocular inflammation in lipopolysaccharide (LPS) induced uveitis. Thus, an effective approach for developing supramolecular assemblies as antiinflammatory ophthalmic therapeutics for eye disorders has come up [26].

\section{Nanotherapeutics}

New delivery solutions are offered by nanotechnology as drug encapsulation and also functional drug carriers. While encapsulation of certain drugs at Nano scale present as better solutions, on the other hand, functional drug carriers use nanomaterials to carry drugs to a specific location in the human body $[27,28]$. 


\section{Targeted drug delivery and pathogen detection by cell surface receptors}

These use encapsulated "smart" materials that recognize targets [29]. These smart systems promise to be much more efficient and effective than current alternatives. For example pathogen detection system for Neisseria gonorrhea has reduced recognition time to minutes, when current methods require days.

\section{Nano encapsulation}

Nano-scale polymer particles when layered make the materials hydrophilic hence immune to natural bodily mechanisms. Thus they ensure that drugs are not released until they reach their intended target. SWRI [South West Research Institute] has developed targeted release systems that encompass Nano capsules including novel vaccines, antibiotics, and drug delivery with reduced side effects. At present, targeted delivery of genes and drugs to human liver has been developed by Osaka University in Japan in 2003. Engineered Hepatitis $B$ virus envelope particles were allowed to form hollow nanoparticles displaying a peptide that is indispensable for liver-specific entry by the virus in humans. Future specialized nanoparticles could be engineered to target oral tissues, including cells derived from the periodontium [30].

\section{Nanoparticle tubes enabling targeted drug release}

Sang Bok Lee developed nontoxic hollow, tube-shaped nanostructures having high drug presence and imaging capabilities. With a layer of magnetite inside, allowing them to be tracked with MRI systems. It additionally contains iron oxide as an MRI enhancement contrast agent. Engineered Hepatitis B virus envelope particles form nanoparticles displaying a peptide that is indispensable for liverspecific entry by the virus in humans. Thus, specialized nanoparticles can be engineered to target oral tissues [7].

\section{Capsula in blood}

These are hypothetical radio-controlled drug delivery nanocapsules measuring about 1 micron in diameter including a storage tank, multiple injection ports, and an antenna allowing external radio control which can enable targeted drug release in human bloodstream [24].

\section{Hydro gels}

These can release their encapsulated drug upon heating eg. Gold Nano shells can absorb infrared wavelength specifically to serve heating purpose. When these optically absorbing nanoshells are heated they transfer their heat to the surrounding [24]. This effect causes the release of drugs from within the Nano composite shells. However in order for this drug delivery system to be effective the Nano composite shells must respond to heating i.e., thermal mechanisms.

\section{Magnetically controlled release of drugs}

Magnetically controlling the release of drugs by the help of nanoparticles is another method whereby drug delivery is benefiting from nanotechnology. Magnetic fluid which typically is Iron nanoparticles in both organic and inorganic fluid binds and concentrates the drug at the site of their delivery [31]. However these must be coated with biodegradable, nontoxic and biologically stable materials.

\section{Dendrimers as oral drug carriers}

Few studies have shown that PAMAM dendrimers can permeate across epithelial barriers by a combination of transcellular and paracellular routes and can transiently open tight junctions. This enhances their transport via the paracellular pathway suggesting their potential as oral drug carriers. However, limited data are available for a systematic correlation of the structure of these polymers with their transport across biological barriers, specifically the intestinal epithelia. In this sense, a general trend observed is that cationic PAMAM dendrimers are more toxic than their anionic counterparts, larger dendrimers are more toxic compared to smaller dendrimers of similar surface functionality, and masking cationic residues with noncharged groups improves tolerability of PAMAM dendrimers and their uptake by the epithelial cells $[9,32]$.

\section{Graphene}

Two-dimensional graphene offers interesting electronic, thermal, and mechanical properties that are currently being explored for advanced electronics, membranes, and composites and also for biological and medical applications. Nano-graphene oxide (NGO), i.e., single-layer graphene oxide sheets are soluble in buffers and serum without agglomeration. The NGO sheets are found to be photoluminescent in the visible and infrared regions. The intrinsic photoluminescence (PL) of NGO is used for live cell imaging in the near-infrared (NIR) with little background. Thus, simple physisorption via $\pi$-stacking can be used for loading doxorubicin, a widely used cancer drug onto NGO functionalized with antibody for selective killing of cancer cells in vitro. Owing to its small size, intrinsic optical properties, large specific surface area, low cost, and useful noncovalent interactions with aromatic drug molecules, it's a promising drug delivery material [33].

Gold nanoclusters (GNCs) impregnated onto reduced graphene oxide (RGO) nanosheets have been shown to cross swiftly across HepG2 hepatocarcinoma cell membranes to alter proteins and DNA and transport anticancer molecular drugs, such as doxorubicin (DOX) [34].

An innovative multifunctional drug delivery system using cRGDmodified chitosan/graphene oxide polymer has also been developed. The polymer presented a good ability of cellular imaging and showed an efficient loading of doxorubicin. Thus, it has the ability to target hepatocarcinoma cells, and is $\mathrm{pH}$-responsive and can be efficiently loaded with a number of therapeutic agents for biomedical applications [35]. These results have been confirmed by the pharmacokinetic and bio distribution profile of a nude tumor bearing mice wherein tumor accumulation of RC/GO/DOX polymers was almost three times higher than the others, highlighting the efficacy of the active targeting strategy [36]. Furthermore, the tumor inhibition rate of $\mathrm{RC} / \mathrm{GO} / \mathrm{DOX}$ polymers was $56.64 \%, 2.09$ and 2.93 times higher than that of CS/GO/DOX polymers (without modification) and the DOX solution, respectively. Thus these anti-tumor efficacy results indicated that the tumor growth was better controlled by RC/GO/DOX polymers than the others. Further studies like cell uptake experiments and a cell proliferation analysis demonstrated that the nanoparticles had higher cytotoxicity for HepG2 and SMMC-7721 cells than chitosan/graphene oxide/doxorubicin (CS-GO-DOX) nanoparticles. 
In vivo anti-tumor experiments demonstrated that the GC-GO-DOX nanoparticles inhibit tumors better than the CS-GO-DOX nanoparticles [37].

\section{Supramolecular nanofibers self-assembled from cationic small molecules}

These were derived from re-purposed poly (ethylene teraphthalate) (PET) and used to self-assemble into high aspect ratio supramolecular nanofibers for encapsulation and delivery of anionic antibiotics. The antibiotic piperacillin/tazobactam (PT) has been successfully loaded into the nanofibers through ionic interaction between anionic PT and the cationic nanofibers without loss of the nanofiber features. These PT-loaded nanofibers demonstrated high loading efficiency and sustained delivery for PT [38].

\section{Gene Therapy}

Kool in 1991, pioneered the Nano circle technology. He used the roller circle amplification technique with special DNA copying enzymes and other chemicals and synthesized first DNA molecule (capable of replicating themselves in test tube). Using RIBOZYME which is capable of cleaving specific drug resistant gene in bacteria, the synthetic nano circle and RNA polymerase in bacterial cell could be used in variety of illness. This concept could make $96 \%$ of targeted genes to stop functioning [39].

\section{Impalefection}

It's a method utilizing nanomaterials such as carbon nano fibers, nanotubes etc. to alter genes. Needle-like nanostructures are synthesized perpendicular to the surface of a substrate. Plasmid DNA containing the gene, intended for intracellular delivery, is attached to the nanostructure surface. A chip with arrays of these needles is then pressed against cells or tissue. Cells that are impaled by nanostructures can express the delivered genes $[39,40]$.

\section{Chromosome Replacement Therapy (CRP)}

Nano robots can replace chromosome from a diseased cell by newly manufactured ones. They can reprogram the cancer cell and produce a healthy tissue. Thus, allowing permanent cure of pre-existing genetic disease [40].

\section{Chromallocyte}

It is a hypothetical Nano robot capable of chromatin replacement of the targeted cell. With the capability of limited vascular surface travel into the capillary bed of the targeted tissue, followed by extravasation, cytopenetration, and complete chromatin replacement in the nucleus of one target cell, and ending with a return to the bloodstream and subsequent extraction of the device from the body, completing the CRT mission. A single lozenge-shaped 69 micron $^{3}$ chromallocyte measures 4.18 microns and 3.28 microns along cross-sectional diameters and 5.05 microns in length [40].

\section{DNA repair machine}

It's an Assembler Built Repair Vessel responsible for genetic maintenance. Its lower pair of arms stretches the DNA coil while the upper arms place regulatory proteins in an intake port [24]. The molecular structure of both DNA and proteins are compared to information stored in the database of a larger Nano computer positioned outside the nucleus and connected to the cell-repair ship by a communications link. Hence, any flaws in proteins and DNA structure are corrected.

\section{Trojan horse}

Intestine targeted drug delivery system has unique advantages in the treatment of inflammatory bowel disease. As a new conception in drug delivery, the Trojan horse system with the synergy of nanotechnology and host cells can achieve better therapeutic efficacy to specific diseases. The feasibility to encapsulate DNA-functionalized gold nanoparticles into primary isolated intestinal stem cells to form an intestinal Trojan horse for gene regulation therapy of inflammatory bowel disease has been proven [41].

\section{Cancer Management}

\section{Anti-cancer drugs}

NITI (Nano interface technology Inc) is developing targeted controlled release of the chemotherapeutic agents which will allow maintaining high concentration of drug around tumor. Nanoparticles of cadmium selenide (quantum dots) glow when exposed to ultraviolet light [42]. When injected, they seep into cancer tumors. The surgeon can thus see the glowing tumor, and use it as a guide for more accurate tumor removal [43]. A cancer vaccine is also underway which works by blocking cancer related tyrosine kinase receptor and its ligand ephrin [44].

The dendrimers that mimic the surface structure of proteins involved in angiogenesis inhibition have been developed for use in antitumor systems. Various studies suggest that arginine dendrimer can be used to suppress or prevent the growth and metastasis of solid tumors $[32,33]$.

Cheng et al. investigated the potential of PAMAM dendrimers as drug carriers of CPT through aqueous solubility studies. Camptothecin (CPT), a plant alkaloid isolated from Camptotheca acuminata is a potent anticancer agent against a wide spectrum of human cancers, including lung, prostate, breast, colon, stomach, bladder, ovarian, and melanoma cancers. However, CPT has an extremely low solubility in aqueous medium, which presents a major challenge during drug formulation in clinical trials. Results showed that the aqueous solubility of CPT was significantly increased by PAMAM dendrimers. In the same context, Goldberg et al. evaluated G3.5 PAMAM dendrimer-SN38 (7-ethyl-10-hydroxycampothecin) conjugates with ester-linked glycine and $\beta$-alanine spacers for their suitability in oral therapy of hepatic colorectal cancer metastases [33].

A formulation of PTX-loaded exosomes with incorporated aminoethylanisamide-polyethylene glycol (AA-PEG) vector moiety to target the sigma receptor, which is overexpressed by lung cancer cells has been tested. The AA-PEG-vectorized exosomes loaded with PTX (AA-PEG-exoPTX) possessed a high loading capacity, profound ability to accumulate in cancer cells upon systemic administration, and improved therapeutic outcomes. The combination of targeting ability with the biocompatibility of exosome-based drug formulations offers a powerful and novel delivery platform for anticancer therapy [45]. 


\section{Tissue Engineering}

Nanotechnology can help to engineer/reproduce/repair any tissue. Tissue engineering makes use of artificially stimulated cell proliferation by using suitable nanomaterial-based scaffolds and growth factors. Tissue engineering will eventually replace today's conventional treatments, such as transplantation of organs or artificial implants. Stem cells are carried by nonorobot carriers and implanted into specific site where organ develops [46].

Few studies suggest that ALN-PAMAM-COOH is highly promising as a restorative biomaterial for in situ remineralization of human tooth enamel. Another example is the work of Chen et al. who investigated the effect of G3.0 PAMAM dendrimers with carboxylic acid (PAMAM$\mathrm{COOH}$ ) on crystal growth on etched enamel. Their study showed that PAMAM-COOH can function as an organic template on the demineralized enamel surface to induce the formation of HAP crystals with the same structure, orientation, and mineral phase of the intact enamel in relatively short time [33].

Synthesis of PAMAM collagen-mimetic conjugates and their resultant complexation with metal cofactors such as $\mathrm{Cu}^{2+}$ and $\mathrm{Ni}^{2+}$ has been attributed by some studies to have extraordinary biological properties mimicking collagen [33].

\section{Discussion}

\section{Magnetic particle targeted wnt signalling}

UM206 is a synthetic peptide and ligand for the Wnt receptor Frizzled. Attachment of UM206 to magnetic nanoparticles (MNP) enables the ligand-MNP complex to be manipulated using magnetic fields, allowing control of Frizzled stimulation. Using this approach, Wnt signaling was activated in hMSC which resulted in Frizzled clustering, $\beta$-catenin translocalization and activation of TCF/LEF responsive transcription. During osteogenesis, UM206-MNP initiated localized mineralized matrix formation. Injection and magnetic stimulation of UM206-MNP-labeled MSC in ex vivo chick femurs resulted in increased mineralization which acted synergistically with addition of bone morphogenic protein 2 (BMP2) releasing microparticles. As this facilitates external control over signal transduction, conjugated MNP technology has applications both as a research tool and for regulating tissue formation in clinical cell therapies [47].

\section{Bone replacement materials and implants}

Hydroxyapatite nanoparticles are being used to treat bone defects are such as Ostim $^{\circ}$ (Osartis GmbH, Germany) HA, VITOSS ${ }^{\circ}$ (Orthovita, Inc., USA) HA + TCP and NanOss ${ }^{\text {Txt }}$ (Angstrom Medica, USA) HA. Nanotechnology has enabled the creation of new surfaces, visual effects and overall changes that are beneficial [48]. Sus Tech GmbH (Darmstadt, Germany) has worked on NANO-APATITE and other biocomposites for the surface of tooth and bone prosthesis [46]. Mostly, the use of artificial implants has problems because the surface is not hard enough and does not interact with tissues, so by making the surface tougher and more interactive, the life of the implant is extended. Scientists are developing a new generation of Nano scale bio structures that solve some of the problems related to the repair and replacement of tissues in the body (the most common problems are immune rejection and invasive surgery). Nano-technology offers new biocompatible nanomaterial and coatings that should increase the adhesion, durability and lifespan of implants. In the case of bone repair, calcium phosphate apatite (CPA) and hydroxyapatite (HAP), for instance, are two Nano ceramic materials that can be used since they have the ability to conform to and bond with bone. Bio-resorbable materials have the advantage of having the capacity to disintegrate without human intervention [49]. For instance, these nanomaterials can be used in sutures and orthopedic fixation devices but, more importantly, they could be used as temporary implants, which would avoid subsequent surgery owing to their biodegradable nature.

\section{Other products}

Anti-pathogenic nanoparticles incorporated in clothing and masks, medical appendages providing instantaneous healing, hemostatic biodegradable nanofibres, silk nanofibres with antimicrobial properties, Nano crystalline silver particles as dressing for wounds, Nano carriers targeting bone, nano needles, nano tweezers and a flesh welder are few other nanomedicinal applications $[50,51]$.

\section{Conclusion}

New nanostructured vaccines could eliminate hazards of conventional vaccine development and use, which rely on viruses and bacteria. Nanotubules that act like tiny straws could conceivably take up drug molecules and release them slowly over time. A Surgical knife made of microstructured-silicon which is nonmagnetic and biocompatible with a chemically inert diamond-layered tip could perhaps make sharper incisions with a lower penetration pressure [46].

Hence the mushrooming nanotechnology has engulfed medical technology and contributed in its upliftment in endless ways and even promises many unrealistic dreams but how far it has touched dentistry will be updated in the upcoming article.

\section{Acknowledgements}

It is my pleasant privilege and honor to express my sincere and heartfelt gratitude towards Dr. A.T. Prakash and Dr. Sumrrita Saroch for their selfless support throughout.

\section{References}

1. Goddard WA (2003) Handbook of nanoscience, engineering, and technology. CRC Press, USA.

2. Generalov M (2007) Nanomaterials in the chemical industry. Chem Petr Eng 43: 376-384

3. Giubileo F, Martucciello N, Di Bartolomeo A (2017) Focus on graphene and related materials. Nanotechnology 28: 410201.

4. http://nanofutures.info/event/nanomaterials-industry-2014

5. Vlachy J (2017) The value of innovation in nanotechnology. Engineering Economics 28: 535-541.

6. Roco M (2005) International Perspective on Government Nanotechnology Funding in 2005. J Nanopart Res 7: 707-712.

7. Kennedy J (2008) Nanotechnology: The future is coming sooner than you think. In presenting futures. The yearbook of nanotechnology in society. Springer Dordrecht, The Netherlands

8. Gupta J (2011) Nanotechnology applications in medicine and dentistry. J Investig Clin Dent 2: 81-88.

9. Pandita D, Poonia N, Kumar S, Lather V, Madaan K (2014) Dendrimers in drug delivery and targeting: Drug-dendrimer interactions and toxicity issues. J Pharm Bioallied Sci 6: 139-150.

10. Shaalan M, Saleh M, El-Mahdy M, El-Matbouli M (2016) Recent progress in applications of nanoparticles in fish medicine: A review. Nanomedicine 12: $701-710$ 
11. Gambhir R, Sogi G, Nirola A, Sekhon T, Kakar H, et al. (2013) Nanotechnology in dentistry: Current achievements and prospects. J Orofac Sci 5: 9.

12. Verma S, Prabhat K, Goyal L, Rani M, Jain A (2010) A critical review of the implication of nanotechnology in modern dental practice. Natl J Maxillofac Surg 1: 41.

13. Chandki R, Kala MNK, Brigit B, Banthia P, Banthia R (2012) Nanodentistry: Exploring the beauty of miniature. J Clin Exp Dent 4 : e119-e124.

14. Smith GBW (2009) Nanoprobe crosses blood-brain barrier to image and treat tumor. MRS Bulletin 34: 711-712.

15. Cheng K, Cheng Z (2012) Near infrared receptor-targeted nanoprobes for early diagnosis of cancers. Current Medicinal Chemistry 19: 4767-4785.

16. Mukherjee A, Shim Y, Song JM (2015) Quantum dot as probe for disease diagnosis and monitoring. Biotechnol J 11: 31-42.

17. Fernandes T (2014) Urine Test Screens Patients for Blood Clots. Clinical Omics 1: 7- 26.

18. Zey M (2017) The future factor (1st edn.). Routledge, UK.

19. Hannestad J (2013) Fluorescence in bio-inspired nanotechnology [electronic resource]: First as probe, then as function. Springer, New York, USA.

20. Kikuchi M (2007) Status and future prospects of biomedical engineering: A Japanese perspective. Biomed Imaging Interv J 3: 25-29.

21. BelBruno J (2013) Nanomaterials in sensors. Nanomaterials 3: 572-573.

22. Henry C (2002) Noninvasive glucose monitoring. Chem Eng News 80: 41-43.

23. Saenz A (2010) Nanotechnology 'tattoos' to help diabetics track glucose levels. Singularity University, USA.

24. https: //www.foresight.org/Nanomedicine/Gallery/Captions

25. Liu M, Zheng S, Zhang X, Guo H, Shi X, et al. (2018) Cerenkov luminescence imaging on evaluation of early response to chemotherapy of drug-resistant gastric cancer. Nanomedicine: Nanotechnology, Biology and Medicine 14: 205-213.

26. Yu X, Zhang Z, Yu J, Chen H, Li X (2018) Self-assembly of a ibuprofenpeptide conjugate to suppress ocular inflammation. Nanomedicine: Nanotechnology, Biology and Medicine 14: 185-193.

27. Yan L, Yang Y, Zhang W, Chen X (2014) Advanced materials and nanotechnology for drug delivery. Adv Mater 26: 5533-5540.

28. Kuzma J, Priest S (2010) Nanotechnology, risk, and oversight: Learning lessons from related emerging technologies. Risk Analysis 30: 1688-1698.

29. Vadlamani L (2010) Nanobots as therapeutic devices. SSRN Electronic Journal 5: 1-5.

30. Vo-Dinh $\mathrm{T}(2007)$ Nanotechnology in biology and medicine. CRC Press Boca Raton, USA.

31. Wei K, Yang H, Huang HL (2012) Potential of magnetic nanoparticles for targeted drug delivery. Nanotechnol Sci Appl 73: 231-236.

32. Noriega-Luna B, Godínez L, Rodríguez F, Rodríguez A, Zaldívar-Lelo de Larrea G, et al. (2014) Applications of dendrimers in drug delivery agents, diagnosis, therapy, and detection J Nanomater 1-19.

33. Sun X, Liu Z, Welsher K, Robinson J, Goodwin A, et al. (2008) Nanographene oxide for cellular imaging and drug delivery. Nano Research 1: 203-212.
34. Wang C, Li J, Amatore C, Chen Y, Jiang H, Wang X (2011) Gold nanoclusters and graphene nanocomposites for drug delivery and imaging of cancer cells. Angew Chem Int Ed 50: 11644-11648.

35. Wang C, Chen B, Zou M, Cheng G (2014) Cyclic RGD-modified chitosan/graphene oxide polymers for drug delivery and cellular imaging. Colloids Surf B Biointerfaces 122: 332-340.

36. Wang C, Li Y, Chen B, Zou M (2016) In vivo pharmacokinetics, biodistribution and the anti-tumor effect of cyclic RGD-modified doxorubicin-loaded polymers in tumor-bearing mice. Colloids Surf B Biointerfaces 146: 31-38.

37. Wang C, Zhang Z, Chen B, Gu L, Li Y, et al. (2018) Design and evaluation of galactosylated chitosan/graphene oxide nanoparticles as a drug delivery system. J Colloid Interface Sci 516: 332-341.

38. Liu S, Fukushima K, Venkataraman S, Hedrick J, Yang Y (2018) Supramolecular nanofibers self-assembled from cationic small molecules derived from repurposed poly (ethylene teraphthalate) for antibiotic delivery. Nanomedicine: Nanotechnology, Biology and Medicine 14: 165-172.

39. Schwartz M (2002) Nanocircles act as Trojan house to shut down disease causing genes. Stanford report.

40. Frietas RA (2007) The ideal gene delivery vector: Chromallocytes, cell repair nanobots for chromosome replacement therapy. Journal of Evolution and technology 6: 1-97.

41. Peng H, Wang C, Xu X, Yu C, Wang Q (2015) An intestinal Trojan horse for gene delivery. Nanoscale 7: 4354-4360.

42. Ashfaq U, Riaz M, Yasmeen E, Yousaf M (2017) Recent advances in nanoparticle-based targeted drug-delivery systems against cancer and role of tumor microenvironment. Crit Rev Ther Drug Carrier Syst 34: 317-353.

43. Zwick E (2001) Receptor tyrosine kinase signalling as a target for cancer intervention strategies. Endocrine Related Cancer 8: 161-173.

44. Pugiliese G (2008) Nanotechnology: Cancer risk.

45. Kim M, Haney M, Zhao Y, Yuan D, Deygen I, et al. (2018). Engineering macrophage-derived exosomes for targeted paclitaxel delivery to pulmonary metastases: in vitro and in vivo evaluations. Nanomedicine: Nanotechnology, Biology and Medicine 14: 195-204.

46. Roco MC, Williams S, Alivisatos P (2000) The interagency working group on nanoscience, engineering and technology - Brochure. Springer, Dordrecht.

47. Rotherham M, Henstock J, Qutachi O, El-Haj A (2018) Remote regulation of magnetic particle targeted Wnt signaling for bone tissue engineering. Nanomedicine: Nanotechnology, Biology and Medicine 14: 173-184.

48. Walmsley GG, McArdle A, Tevlin R (2015) Nanotechnology in bone tissue engineering. Nanomedicine $\mathbb{X}$ : Nanotechnology, biology, and medicine 11: 1253-1263.

49. Thakral GK, ThakralR, Sharma N, Seth J, Vashisht P (2014) Nanosurface: The future of implants. J Clin Diagn Res 8: ZE07-ZE10.

50. Hamdan S, Pastar I, Drakulich S, Dikici E, Tomic-Canic M, et al. (2017) Nanotechnology-driven therapeutic interventions in wound healing: potential uses and applications. ACS Cent Sci 22: 163-175.

51. Rajkumar SRJ, Nadar MSAM, Selvakumar PM (2017) Nanotechnology in wound healing: A review. Glob J Nanomed 3: 1-4. 